1987, 36, 2

УдК 535.33

Ннна РЕБАНЕ

\title{
ПОВЫШЕНИЕ РАЗРЕШАЮЩЕЙ СПОСОБНОСТИ МЕТОДА ФОТОВЫЖИГАНИЯ СПЕКТРАЛЬНОГО ПРОВАЛА ПУТЕМ ДВУХСТУПЕНЧАТОГО ВЫЖИГАНИЯ
}

Inna REBANE. SPEKTRAALSE FOTOSALKAMISE MEETODI LAHUTUSVOIME SUURENDAMINE Inna REBANE. THE INCREASE OF THE RESOLVING POWER OF THE METHOD OF SPECTRAL HOLE-BURNING

\section{(Представил В. Хижняков)}

Показано, что использование двухступенчатого процесса фотовыжигания провала может существенно повысить разрешающую способность метода.

Рассмотрим фотовыжигание спектрального провала двумя импульсами, центральные частоты которых сдвинуты относительно друг друга на частоту $\omega_{1}$. Функцию, описывающую временное поведение такого выжигающего поля, выберем в виде суперпозиции двух экспоненциально затухающцх импульсов

$g(t)=\left\{\begin{array}{l}0, \text { если } t<0, \\ \sqrt{\Delta} \exp (-\Delta t / 2)\left[\exp \left(-i \omega_{0} t\right)+\exp \left(-i\left(\omega_{0}+\omega_{1}\right) t\right)\right], \text { если } t \geqslant 0 .\end{array}\right.$

В формуле (1) $\Delta$ - спектральная ширина импульсов (полная ширина на половине высоты), $\omega_{0}$ и $\omega_{0}+\omega_{1}-$ центральные частоты импульсов. Частотное распределение импульсов состоит из двух лоренцианов и интерференционного члена

$$
\begin{aligned}
& |g(\omega)|^{2}=\frac{\Delta}{x^{2}+\Delta^{2} / 4}+\frac{\Delta}{\left(x-\omega_{1}\right)^{2}+\Delta^{2} / 4}+ \\
& +\frac{\Delta}{\omega_{1}^{2}+\Delta^{2}}\left[\frac{\Delta^{2}-2 \omega_{1} x}{x^{2}+\Delta^{2} / 4}+\frac{\Delta^{2}+2 \omega_{1}\left(x-\omega_{1}\right)}{\left(x-\omega_{1}\right)^{2}+\Delta^{2} / 4}\right],
\end{aligned}
$$

где $g(\omega)=\int g(t) \exp (i \omega t) d t, x=\omega-\omega_{0}$.

Изменение функции неоднородного распределения (ФНР) @ $\left(\Omega_{01}\right)$ частот оптического перехода $\Omega_{01}$ двухуровневых систем в результате прохождения световых импульсов через селективную фотохромную среду можно записать сле:іующим образом [ $\left.{ }^{1}\right]$ :

$$
\varrho\left(\Omega_{01}\right)=\varrho_{0}\left(\Omega_{01}\right)\left[1-P_{1}\left(\Omega_{01}\right)\right],
$$

где $\varrho_{0}\left(\Omega_{01}\right)$ - первоначальная ФНР. Вероятность $P_{1}\left(\Omega_{01}\right)$, определяющая провал в ФНР $\mathrm{Q}\left(\Omega_{01}\right)$, имеет вид $\left[{ }^{2}\right]$ :

$$
P_{1}\left(\Omega_{01}\right) \sim \alpha_{1} \int_{-\infty}^{\infty} d \omega|g(\omega)|^{2} \varkappa\left(\Omega_{01}, \omega\right) .
$$

Здесь $\alpha_{1}$ - вероятность превращения возбужденной на уровень 1 системы и $x\left(\Omega_{01}, \omega\right)$ - однородный спектр поглощения. 
В простейшей модели $x\left(\Omega_{01}, \omega\right)=\gamma_{1} /\left[\left(\omega-\Omega_{01}\right)^{2}+\frac{\gamma_{1}^{2}}{4}\right]$, где $\gamma_{1}$ константа энергетической релаксации возбужденного уровня 1, вероятность $P_{1}\left(\Omega_{01}\right)$ имеет следующий вид:

$$
\begin{gathered}
P_{1}\left(\Omega_{01}\right) \sim \alpha_{1}\left\{\frac{\Delta+\gamma_{1}}{y^{2}+\left(\Delta+\gamma_{1}\right)^{2} / 4}+\frac{\Delta+\gamma_{1}}{\left(y-\omega_{1}\right)^{2}+\left(\Delta+\gamma_{1}\right)^{2} / 4} H,\right. \\
\left.+\frac{\Delta}{\omega_{1}^{2}+\Delta^{2}}\left[\frac{\Delta\left(\Delta+\gamma_{1}\right)-2 \omega_{1} y}{y^{2}+\left(\Delta+\gamma_{1}\right)^{2} / 4}+\frac{\Delta\left(\Delta+\gamma_{1}\right)+2 \omega_{1}\left(y-\omega_{1}\right)}{\left(y-\omega_{1}\right)^{2}+\left(\Delta+\gamma_{1}\right)^{2} / 4}\right]\right\},
\end{gathered}
$$

где $y=\Omega_{01}-\omega_{0}$.

Из формулы (5) следует, что в рассматриваемом случае выжйаются либо два провала с максимумами на частотах $\Omega_{01}=\omega_{0}$ и $\Omega_{01}=$ $=\omega_{0}+\omega_{1}$ и ширинами $\Delta+\gamma_{1}$ (см. рисунок), либо один провал с максимумом на частоте $\Omega_{01}=\omega_{0}+\omega_{1} / 2$ (два провала сливаются в один).

Однако есть возможность сузить выжигаемые данными импульсами провалы и тем самым повысить разрешающую способность методики. Для этого надо использовать определенный двухступенчатый процесс фотовыжигания провала в трехуровневой системе [3]. В этом процессе рассматриваемые два импульса используются для частотно-селективного возбуждения в области неоднородно уширенной линии поглощения $0 \rightarrow 1$, поглощение второго предельно короткого импульса ( $\delta$-импульса) возбужденным состоянием $(1 \rightarrow 2)$ приводит к фотоионизации и выжиганию провала. В этом случае вероятность $P_{2}\left(\Omega_{01}\right)$, определяющая провал в $Ф Н \mathrm{Q} \mathrm{Q}\left(\Omega_{01}\right)$ частот оптического перехода $0 \rightarrow 1$, имеет следующий вид $\left[{ }^{3}\right]$ :

$$
P_{2}\left(\Omega_{01}\right) \sim \alpha_{2} \int_{T}^{\infty} d t^{\prime} \int_{-\infty}^{T} d t_{2} d t_{2}^{\prime} g^{*}\left(t_{2}^{\prime}\right) g\left(t_{2}\right) F\left(t^{\prime}, T, T, t_{2}, t_{2}^{\prime}\right)
$$

Здесь $\alpha_{2}$ - вероятность превращения возбужденной на уровень 2 системы, $T$ - момент времени прохождения через центр системы $\delta$-импуль$\mathrm{ca}, F\left(t^{\prime}, t_{1}, t_{1}{ }^{\prime}, t_{2}, t_{2}^{\prime}\right)$ - корреляционная функция трехуровневой системы, которая в простейшей модели (релаксационные процессы на первом и на втором возбужденном уровне описываются константами энергетической релаксации $\gamma_{1}$ и $\gamma_{2}$ ) имеет следующий вид:

$$
\begin{aligned}
& F\left(t^{\prime}, t_{1}, t_{1}^{\prime}, t_{2}, t_{2}^{\prime}\right)=C \exp \left[-\gamma_{2} t^{\prime}+i \Omega_{12}\left(t_{1}-t_{1}^{\prime}\right)+\right. \\
& \left.+\left(\gamma_{2}-\gamma_{1}\right)\left(t_{1}+t_{1}^{\prime}\right) / 2+i \Omega_{01}\left(t_{2}-t_{2}^{\prime}\right)+\gamma_{1}\left(t_{2}+t_{2}^{\prime}\right) / 2\right] .
\end{aligned}
$$

Подставляя формулы (1) и (7) в формулу (6) и ннтегрируя по формуле (6), получаем:

$$
\begin{aligned}
& P_{2}\left(\Omega_{01}\right) \sim \alpha_{2}\left\{\left[\exp (-\Delta T)+\exp \left(-\gamma_{1} T\right)-2 \cos (y T) \exp \left(-\left(\Delta+\gamma_{1}\right) T / 2\right)\right] /\right. \\
& /\left[y^{2}+\left(\gamma_{1}-\Delta\right)^{2} / 4\right]+\left[\exp (-\Delta T)+\exp \left(-\gamma_{1} T\right)-\right. \\
& \left.-2 \cos \left(\left(y-\omega_{1}\right) T\right) \exp \left(-\left(\Delta+\gamma_{1}\right) T / 2\right)\right] /\left[\left(y-\omega_{1}\right)^{2}+\left(\gamma_{1}-\Delta\right)^{2} / 4\right]+ \\
& \quad+\left[( \operatorname { e x p } ( ( i y - \Delta / 2 ) T ) - \operatorname { e x p } ( - \gamma _ { 1 } T / 2 ) ) \left(\exp \left(i\left(\omega_{1}-y\right)-\Delta / 2\right) T-\right.\right. \\
& \left.\left.\left.-\exp \left(-\gamma_{1} T / 2\right)\right) /\left(\left(i y+\left(\gamma_{1}-\Delta\right) / 2\right)\left(i\left(\omega_{1}-y\right)+\left(\gamma_{1}-\Delta\right) / 2\right)\right)+c . c .\right]\right\}
\end{aligned}
$$

Из формулы (8) следует (см. также рисунок), что с увеличением времени $T$ между первым и вторым этапами возбуждения провалы монотонно сужаются. Предельная (при $T \rightarrow \infty)$ ширина провалов определяется параметром $\left|\gamma_{1}-\Delta\right|$. В случае $T^{-1},\left|v_{1}-\Delta\right| \ll \gamma_{1}, \Delta$ провалы, описываемые формулой (8), значительно уже, чем в двухуровневом случае (см. формулу (5)). Ширины $\gamma_{1}$ и $\Delta$ компенсируют друг друга. Возникает ситуация, когда в двухуровневом случае отмечается один 


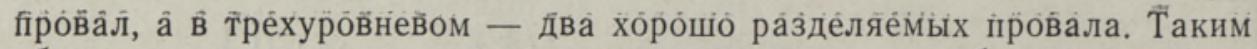
образом, значительно повышается разрешающая способность методики фотовыжигания провалов в $Ф Н Р \varrho\left(\Omega_{01}\right)$ частот оптических переходов.
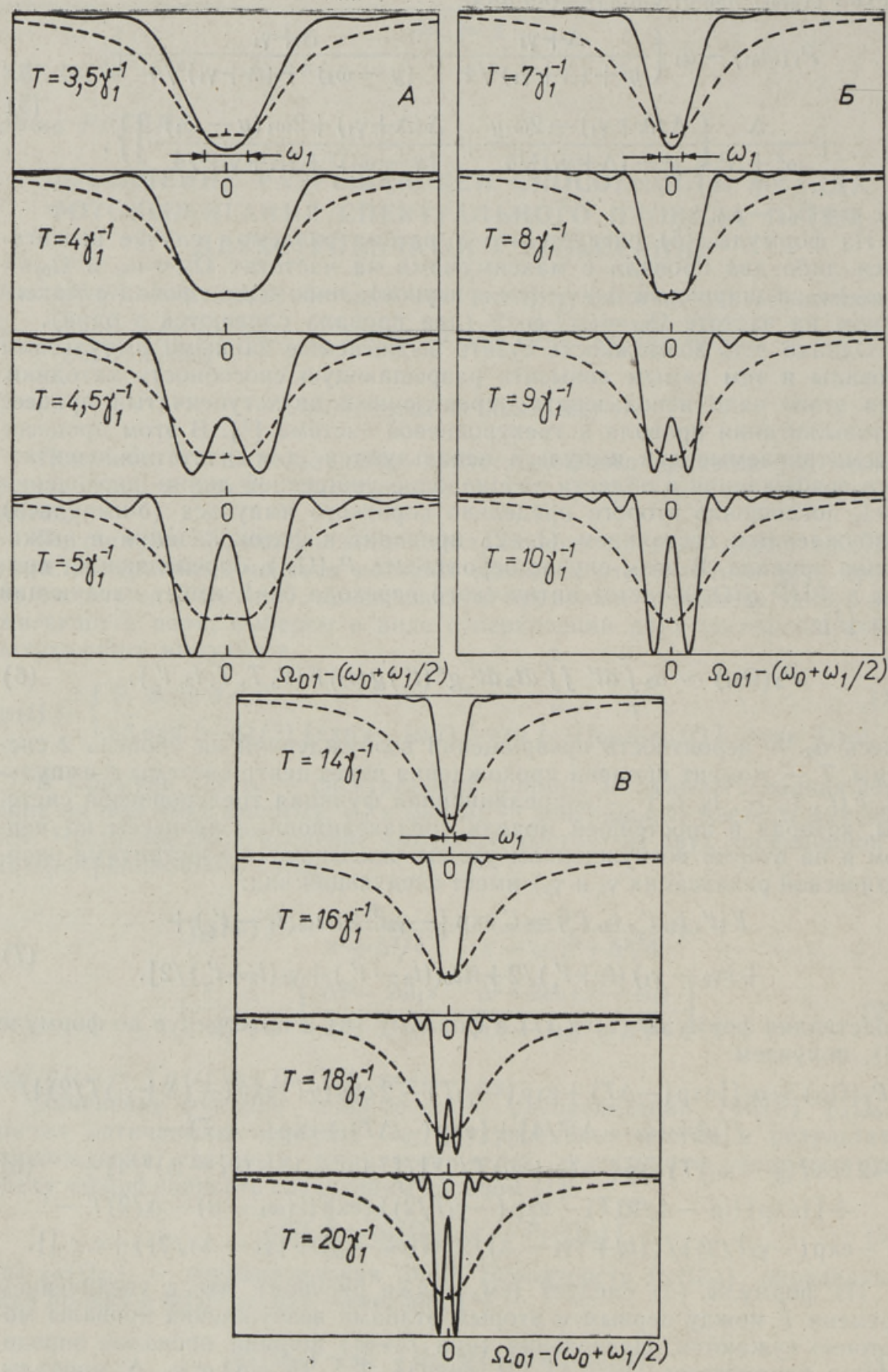

Провалы в ФНР $\varrho\left(\Omega_{01}\right)$ при двухступенчатом (сплошная линия) и одноступенчатом (штриховая линия) процессах. $\left(\Delta=0,99 \gamma_{1}\right)$. A $-\omega_{1}=\gamma_{1} ;$ Б $-\omega_{1}=0,5 \gamma_{1}$; $\mathrm{B}-\omega_{1}=0,25 \gamma_{1}$. 
С̂жение прровалов с увеличением времени $T$ объясняется тем, чито̄ при рассматриваемом двухступенчатом возбуждении провал в ФНР $\varrho\left(\Omega_{01}\right)$ отражает распределение вероятностей нахождения систем на возбужденных уровнях 1 именно в момент времени $T$ : второй импульс застает на уровнях 1 только те центры, которые еще не перешли на основной уровень 0 , т. е. уже прожили некоторое время на уровне 1 и «остыли». Поэтому с ростом времени $T$ наряду с уменьшением глубины провала происходит его сужение.

В заключении отметим, что если усложнить модель модуляционным уширением (фазовой релаксации) первого возбужденного уровня 1, то предельные ширины провалов в $Ф Н \mathrm{P} \varrho\left(\Omega_{01}\right)$ будут определяться параметром $\left|\gamma_{1}-\Delta\right|+\Gamma$, где $\Gamma-$ константа фазовой релаксации, т. е. модуляционное уширение не компенсируется, а прибавляется к прежней ширине.

Автор благодарен К. К. Ребане и В. В. Хижнякову за обсуждение работы.

\section{ЛИТЕРА Т Р Р}

1. Rebane, L. A., Gorokhovskii, A. A., Kikas, J. V. Appl. Phys. B, 29, 235-250 (1982).

2. Ребане И. Изв. АН ЭССР. Физ. Матем., 34, № 4, 438-440 (1985).

3. Ребане И. Изв. АН ЭССР. Физ. Матем., 35, № 3, 296-301. (1986).

Институт физики

Академии наук Эстонской ССР
Поступила в редакцию 13/XI 1986 